\title{
Optical Properties of a Novel Yellow Fluorescent Dopant for use in organic LEDs
}

\author{
Wallace. C.H. Choy ${ }^{1 \#}$, Yao-Shan $\mathrm{Wu}^{2}$, Chin H. Chen ${ }^{2}$, K.W. Cheah ${ }^{3 \#}$ \\ ${ }^{1}$ Department of Electrical and Electronic Engineering, University of Hong Kong, \\ Hong Kong SAR, PRC. \\ ${ }^{2}$ OLED Technology Research Laboratory, Microelectronics \& Information Systems \\ Research Center and Department of Applied Chemistry, \\ National Chiao Tung University, HsinChu, Taiwan. \\ ${ }^{3}$ Department of Physics, Hong Kong Baptist University, Hong Kong SAR, PRC.
}

In this paper, the optical properties of a novel organic, 2,8-di(t-butyl)-5,11-di[4(t-butyl) phenyl]-6,12-dipehnylnaphthacene (tetra(t-butyl)rubrene) have been investigated. Our results show that there are two peaks in the photoluminescence (PL) spectra of tetra(tbutyl)rubrene (TBRb) which are also confirmed in the electroluminescence (EL) spectra. Photo-quenching of the PL intensity is observed when the irradiation time increases. It is shown that oxidation is the dominant reason for photo-quenching. The absolute refractive index and absorption coefficient have also been determined and the results correlate well with the PL results. The results show that TRBb can be a good dopant to achieve the Förster energy transfer and to assist light emission. The optical properties of TBRb are similar to that of rubrene, however, the PL of TBRb is much stronger than that of rubrene. Finally, although crystalline organics have been commonly reported by heating the sample, we report crystallization of TBRb at low temperature $<230 \mathrm{~K}$ when the TBRb film is in amorphous form before cooling.

PACS no.: 78.47.+p; 78.55.-m; 81.05.Lg; 85.60.-q

\# Email: chchoy@eee.hku.hk, kwcheah@hkbu.edu.hk, Fax: (852) 2559-8738 (HKU) 


\section{Introduction}

Archetypical 5,6,11,12-tetraphenylnaphthacene (rubrene) has been widely investigated for more than three decades. The optical properties of solution-form rubrene (Rb) have been widely studied and used to investigate the quantum yields of singlet oxygen production and the effectiveness of singlet oxygen quenchers [1-4]. On the other hand, $\mathrm{Rb}$ in solid state has also been utilized in organic optoelectronic devices. In the case of organic light emitting diode (OLED), Rb has been considered as an important yellow fluorescent dye $[5,6]$. Yellow is one of the more sensitive colours to human eyes and generally preferred by most users, hence, a stable and efficient yellow is very desirable. $\mathrm{Rb}$ also has other interesting features such as resisting concentration quenching [7], improving the electro-luminescent efficiency of OLED [8-10] and enhancing device stability and lifetime $[11,12]$.

However, there is a lack of study in the optical properties of amorphous form Rb [13], particularly in the derivatives of Rb. In this paper, we investigate the optical properties of a novel derivative of $\mathrm{Rb}$ which is 2,8-di(t-butyl)-5,11-di[4(t-butyl)phenyl]-6,12dipehnylnaphthacene, hitherto named as tetra(t-butyl)rubrene (TBRb). We will first discuss the absolute refractive index and absorption coefficient of TBRb. From the absorption spectrum, suitable excitation energy can be found for photoluminescence (PL) measurement. The absorption properties are critical for utilizing an emitting assist dopant in achieving efficient Förster energy transfer and thus improving the luminescent efficiency [13-15]. Meanwhile, both the refractive index and absorption coefficient are the key parameters in designing of the material structures in optoelectronic devices 
particularly in microcavity devices [16,17]. Besides, low temperature and room temperature PL was studied. Luminescence lifetime of TBRb in various temperatures will also be discussed. Finally, crystallization of TBRb at low temperature will be reported.

\section{Experimental Description}

$\mathrm{Rb}$ was purchased from Aldrich Chemical Co., Inc. and was used without further purification. TBRb was synthesized following the literature described elsewhere [18]. $\mathrm{Rb}$ and $\mathrm{TBRb}$ thin films were prepared by thermal evaporation using Edwards 306. The coating rate is about $1 \AA / \mathrm{s}$. The thickness of TBRb and Rb were monitored in situ using a quartz crystal sensor and further calibrated by a profilometer (Tencor Alpha-step 500) and Woollam V-VASE ellipsometer. Quartz plate and silicon wafer were used as the substrate for film formation. The absorbance was measured using Varian Cary 100 Scan Spectrometer with the correction of the substrate. The absolute reflective index and absorption coefficient were determined using Woollam V-VASE ellipsometer with AutoRetrader. He-Cd laser with 325nm line was used for PL measurement and the luminescence was collected into a double $1 / 4$ meter monochromator. The light was then detected by a photomultiplier tube (PMT) with a response time of $\sim 1 \mathrm{~ns}$. The sample was placed in a cryostat for low temperature PL measurement and the temperature was cooled down to as low as $11 \mathrm{~K}$. For lifetime measurement, the light source was $\mathrm{N}_{2}$ pulse laser with a wavelength of $337 \mathrm{~nm}$. The signal was then amplified by a high speed preamplifier with rise time $<1$ ns and measured by a $600 \mathrm{MHz}$ digital storage oscilloscope. 
The morphology of the sample was investigated using Scanning Electron Microscopy (SEM) and X-ray Ray Diffraction (XRD).

\section{Results and Discussion}

Fig. 1 shows the structure of $\mathrm{Rb}$ and TBRb. Four $t$-butyl groups are attached to $\mathrm{Rb}$ molecular structure to form TBRb. In order to have an efficient photo-excitation for PL, the absorption spectrum of TBRb film was first investigated. As shown in Fig. 2, the absolute refractive index and absorption coefficient with the wavelength in the range from 300nm to $800 \mathrm{~nm}$ were determined. TBRb has been grown on silicon wafer for investigating absolute optical properties because silicon has high refractive index to increase the detected signal. Besides, since the absorption coefficient of silicon is high in visible light, the reflection of the light from the bottom of the substrate can be eliminated which if happens would result in a disturbance signal to interfere the results of ellipsometry. The real part of refractive index peaks at 316nm with a value of 1.776 and gradually decreases when wavelength increases. There are also two resolvable shoulder peaks at about 496nm and 540nm. Similar features were also obtained for the absorption coefficient as shown in Fig. 2. The highest absorption peak with a value of $47,986 \mathrm{~cm}^{-1}$ is obtained at the wavelength of $304 \mathrm{~nm}$ and two small but clear peaks are at $496 \mathrm{~nm}$ and $524 \mathrm{~nm}$. The spectrum of absorption coefficient is in a very good agreement with the absorbance of TBRb (the spectra not shown here) coated on quartz plate with the same film thickness. By using two different techniques (ellipometry and transmission spectrometry) and two different substrates (silicon wafer and quartz plate), we conclude that the absorption features are from the TRBb instead of from the substrate. 
The absorption peaks of TBRb at $496 \mathrm{~nm}$ and $524 \mathrm{~nm}$ are very similar to that of $\mathrm{Rb}$ $[13,15]$. The absorption region between $450 \mathrm{~nm}$ and 550nm of both $\mathrm{Rb}$ and TBRb well overlap with the luminescent spectrum of some good electron transport organics such as tris (8-hydroxyquinolinolato) aluminum (Alq3) [13,15]. Together with the lowest unoccupied molecular orbital (LUMO) and the highest occupied molecular orbital (HOMO) $(-3.2 \mathrm{eV}$ and $-5.49 \mathrm{eV})$ of TBRb [18] existed between that of Alq3 (typically $3 e V$ and $-5.7 e V)$, the injected electrons and holes in Alq3 can be efficiently transfer to TBRb. In addition, the excitation energy of TBRb molecules can also efficiently transfer to some red organic dye such as 4-Dicyanomethylene-2-methyl-6-[2-2(2,3,6,7-tetrahydro1H,5H-benzo[ij]quinolizin-8-yl]-4H-pyran (DCM2) and thus enhance the red colour luminescence efficiency. It is because the LUMO and HOMO $(-3.11 \mathrm{eV}$ and 5.26eV [14]) of DCM2 are close to that of TBRb. As a consequence, the absolute absorption coefficient and refractive index have been determined which is important for designing the material system and layer structures [16] of organic LEDs. Besides, the results show that TRBb can be a good dopant to achieve efficient Förster energy transfer and to assist light emission. It should be noted that since absorption is stronger in short wavelength region, we used 325nm source for PL measurement.

The PL of TBRb and $\mathrm{Rb}$ at various temperatures from $300 \mathrm{~K}$ to $11.5 \mathrm{~K}$ are shown in Fig. 3. The PL peak intensity of TBRb is generally higher than that of $\mathrm{Rb}$ and the peak wavelengths are approximately 10nm longer than that of $\mathrm{Rb}$. The red-shifted peak wavelength of TBRb can also be obtained in diluted solution form [18], i.e. the feature is 
not due to the solid state effects. Recently, theoretical work has been carried out in tetracene, RB and TBRb using commercial model Gaussian 98 followed by density functional theory to determine the Kohn-Sham molecular orbitals by C.F. Lo’s group [19]. There is a trend that the energy difference between LUMO and HOMO reduces (i.e. red shift) in a sequence from tetracene to $\mathrm{Rb}$ and then TBRb [20]. The change of the molecule structure and thus the orbit energies of the molecules may be one of the reasons for the red shift of the PL peak wavelength of TBRb as compared to Rb. Concerning the PL intensity, the four t-butyl groups of TBRb could be considered as spacer, as shown in Fig. 1, to increase the separation between TRBb molecules as compared to that of $\mathrm{Rb}$ molecules, it therefore reduces the quenching effect under the irradiation of He-Cd laser. As a consequence, the PL intensity of TBRb is higher than that of Rb. A brighter organic LED can be therefore obtained by TBRb, which has also been experimentally demonstrated recently [18]. In Rb, the energy gap between the first two singlets and that between two triplets are approximately $2.5 \mathrm{eV}$ [21]. However, from the lifetime of amorphous form $\mathrm{TBRb}$ and $\mathrm{Rb}$ (will be discussed later), the magnitudes are less than 15ns at various wavelengths over the PL spectrum (see Fig. 6). Therefore, it is believed that the PL shown in Fig. 3 primarily corresponds to singlet transitions. It should be noted that the two-peak spectrum of EL [18] and PL of TBRb is not due to aggregation but is the inherent emission characteristics of the molecule because the two peaks PL spectrum can also be obtained in a very dilute $1.8 \times 10^{-9} \mathrm{M}$ TBRb solution in which the aggregation between molecules can be ignored. The TBRb solution is prepared by dissolving TBRb in tubrene. Moreover, as in Fig. 3, the stronger peak at the blue side will undergo a red shift by about $10 \mathrm{~nm}(\sim 3.7 \mathrm{meV})$ when the temperature reduces from 
$300 \mathrm{~K}$ to $150 \mathrm{~K}$ in both $\mathrm{Rb}$ and TBRb. This may be due to the phase change of the materials.

Furthermore, the PL intensity is reduced when the irradiation time increases due to photoquenching. In order to elucidate the reason, the decay of PL peak at room atmosphere and $1 \times 10^{-5}$ mbar vacuum has been investigated as shown in Fig. 4. It should be noted that the He-Cd laser irradiation is permanently switched on during the measurement. For the case of room atmosphere, the PL peak intensity at the wavelength of $575 \mathrm{~nm}$ exponentially reduces to almost close to zero after 1500 sec of irradiation. However, when the PL is measured in $1 \times 10^{-5}$ mbar vacuum, a stable and constant emitted light intensity can be obtained although the intensity also exponentially decays in the first 400sec. It is reasonable to assume that the decay in the vacuum case is due to some oxygen present on the surface of the sample, which forms oxide on the TBRb sample. From the results above, the reduction of emission intensity may be due to the oxidation of TBRb.

Both TBRb and Rb display the presence of two lifetimes in the transient PL spectrum. They show similar short lifetime at about 5-6ns while the long one is greater than 30ns. The TBRb transient PL at the wavelength of 587nm is shown in Fig. 5. The lifetime of the exponential decay with slope A is $6.6 \mathrm{~ns}$ and that with slope B is $31.7 \mathrm{~ns}$. The short lifetime decay at wavelengths over the whole PL spectrum of TBRB is summarized in Fig.6. Generally, the lifetime increases when temperature reduces because the vibronic transitions and non-radiative transition reduces at the lower temperature. In addition, the 
lifetime increases as the monitored wavelength of the PL spectrum increases. At $11 \mathrm{~K}$ (filled square in Fig. 6), the lifetime slightly reduces when the wavelength increases from $587 \mathrm{~nm}$ to $625 \mathrm{~nm}$ and then increases again. This may be because of the change of the emission centers from one center with PL peak at 587nm to another one at $625 \mathrm{~nm}$ as shown in Fig. 3. The similar feature can also be obtained in the case of 300K.

While crystalline organic materials usually form after thermal annealing ( $>300 \mathrm{~K})$, we report that the crystallization is also possible at low temperature. Figure 7 shows the crystallization of TBRb after cooling at the temperature $<230 \mathrm{~K}$ and at a pressure of $1 \times 10^{-5}$ mbar. To further investigate the crystallization at low temperature, we have carried out XRD analysis on samples before and after cooling. There is no useful signal for the before-cooled sample, i.e. no crystal forms. However, after cooling the samples at $\mathrm{T}<230 \mathrm{~K}$, clear peaks are obtained at various diffraction angles, i.e. crystal forms after cooling. One possible explanation for the crystallization is that when the temperature increases to room temperature after cooling at $\mathrm{T}<230 \mathrm{~K}$, thermal energy is provided to the amorphous TBRb. The energy compensates the activation energy of TBRb such that TBRb changes from amorphous phase to crystalline phase as shown in Fig. 7. As a consequence, both SEM and XRD show that TBRb crystallized after cooling at low temperature $(\mathrm{T}<230 \mathrm{~K})$.

\section{Conclusion}

In conclusion, the optical properties of $\mathrm{TBRb}$ and $\mathrm{Rb}$ in various temperatures have been investigated including absolute refractive index, absorption coefficient, PL and photon 
lifetime of TBRb. The features of absorption spectrum determined by spectroscopic ellipsometry are in a very good agreement with that obtained by the transmission method. The results show that TBRb can be a good dopant to achieve efficient Förster energy transfer for typical host organics and to assist light emission of popular dyes. Besides, the PL of TBRb is about 5 times higher than that of Rb, i.e. TBRb should be able to contribute to a brighter organic LED. Our results show that the photo-quenching of TBRb is mainly due to oxidation. TBRb similar to Rb have two lifetimes; the short one is $\sim 5 \mathrm{~ns}$ while the long one is $\sim 31 \mathrm{~ns}$. When the monitored wavelength of the PL spectrum increases, the lifetime increases. Meanwhile, the lifetime also increases when temperature reduces. Finally, crystallization of TBRb is observed after cooling at $\mathrm{T}<$ $230 \mathrm{~K}$ and low pressure of $1 \times 10^{-5} \mathrm{mbar}$ when typical crystalline organics are commonly formed at high temperature ( $>300 \mathrm{~K})$.

ACKNOWLEDGEMENT: The authors would like to thank H.H. Fong for XRD measurement. This project is supported by the RGC of Hong Kong Government, UDF of the University of Hong Kong and the FRG of Hong Kong Baptist University. C.H.C. also thanks the Ministry of Education of Taiwan, R.O.C. for supporting his OLED research at NCTU by a grant from PPUAE (91-E-FA04-2-4). 


\section{References:}

1. B.E. Algar, B. Stevens, J. Phys. Chem, 74, 3029 (1970).

2. P.B. Merkel, D.R. Kearns, J. Am. Chem. Soc, 94, 7244 (1972).

3. B. Stevens, J.A. Ors, J. Phys. Chem, 80, 2164 (1976).

4. P.B. Merkel, W.G. Herkstoeter, Chem Phys. Lett, 53, 350 (1978).

5. T. Sano, Y. Hamda, K. Shibata, IEEE J. Sel. Top. Quantum Electron, 4, 34 (1998).

6. Y. Sato, Semiconductors and Semimetals, 64, 209 (2000).

7. Y. Sato, T. Ogata, S. Ichinosawa, Y. Murata, Syn. Met., 91, 103 (1997).

8. T.K. Hatwar, G. Rajeswaran, J. Shi, Y. Hamada, K. Kanno, H. Takahashi, Proceeding of the $10^{\text {th }}$ International Workshop on Inorganic and Organic Electroluminscence (EL’00), Hamamatsu, Japan, 31 (2000).

9. T.H. Liu, C.Y. Iou, C.H. Chen, Appl. Phys. Lett, 83, 5241 (2003).

10. D. J. Fatemi, H. Murata, C.D. Merritt, Z.H. Kafafi, Syn. Met., 85, 1225 (1997).

11. H. Aziz and Z. D. Popovic, Appl. Phys. Lett, 80, 2180 (2002).

12. Z.L. Zhang, X.Y. Jiang, S.H. Xu, T. Nagatomo, O. Omoto, Syn. Met., 91, 131 (1997).

13. H. Mattoussi, H. Murata, C.D. Merritt, Y. Iizumi, J. Kido, Z.H. Kafafi, J. Appl. Phys., 86, 2642 (1999).

14. Y. Hamada, H. Kanno, T. Tsujioka, H. Takahashi, T. Usuki, Appl. Phys. Lett, 75, 1682 (1999).

15. F. Li, J. Lin, F. Feng, G. Cheng, H. Liu, S. Liu, L. Zhang, Z. Zhang, S.T. Lee, Syn. Met., 139, 341 (2003).

16. W.C.H. Choy, E.H. Li, IEEE J. Quantum Electron., 33, 382 (1997).

17. H. L. Tam, R. Huber, K. F. Li, W. H. Wong, Y. B. Pun, S. K. So, K. W. Cheah, Physica E, 17, 448 (2002)

18. Y.S. Wu, T.H. Liu, C.Y. Iou, C.H. Chen, International Conference on the Science and Technology of Emissive Displays and Lighting (EL 2002), Ghent, Belgium, Sept 2326, 273 (2002).

19. H.H. Fong, S.K. So, W.Y. Sham, C.F. Lo, Y.S. Wu, C.H. Chen, Chem. Phys., 289, 119 (2004).

20. C.F. Lo, a private communication.

21. A. Yildiz, P.T. Kissinger, C.N. Reilley, J. Chem. Phys. 49, 1403 (1968).

Figure Captions

Fig. 1 The structure of (a) TBRb and (b) Rb

Fig. 2 The absolute refractive index and absorption coefficient of TBRb thin film grown by thermal evaporator.

Fig. 3 PL of TBRb thin film at different temperatures.

Fig. 4 Photo-quenching of TBRb thin film at room atmosphere and $1 \times 10^{-5} \mathrm{mbar}$.

Fig. 5 Lifetime of the transient PL of TBRb thin film.

Fig. 6 Lifetime of the transient PL of TBRb at $10 \mathrm{~K}$ and room temperature

Fig. 7 Crystallized TBRb (a) SEM picture and (b) XRD result. 


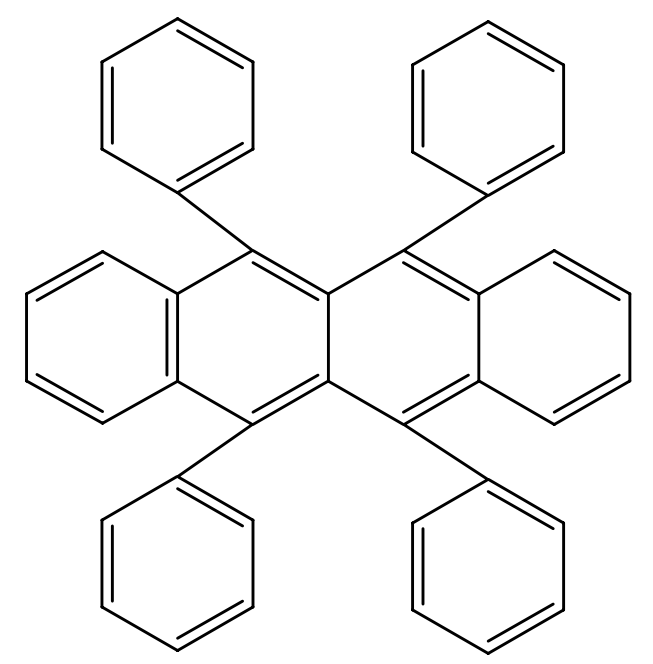

Figure 1a

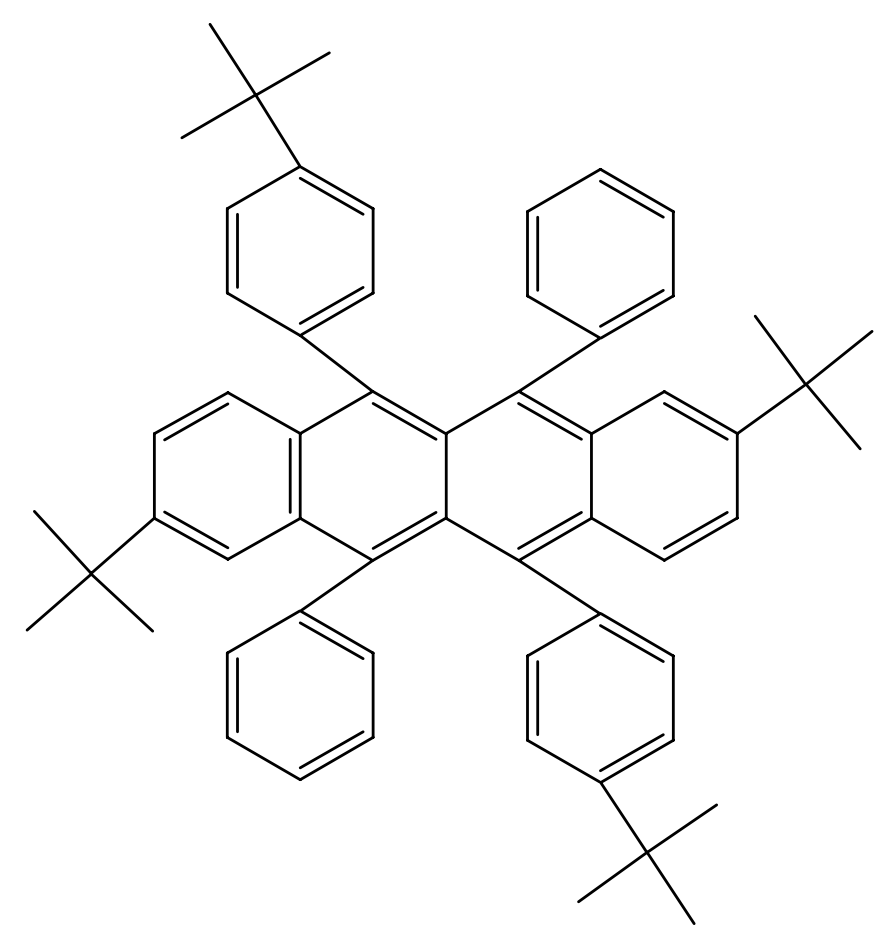

Figure 1b 


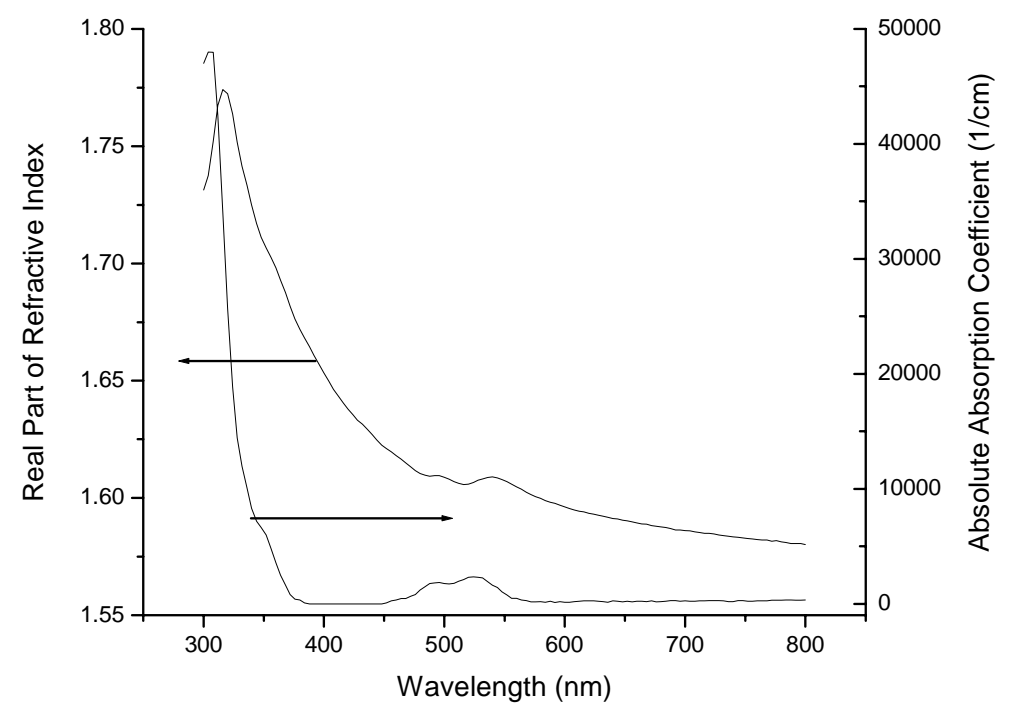

Figure 2 


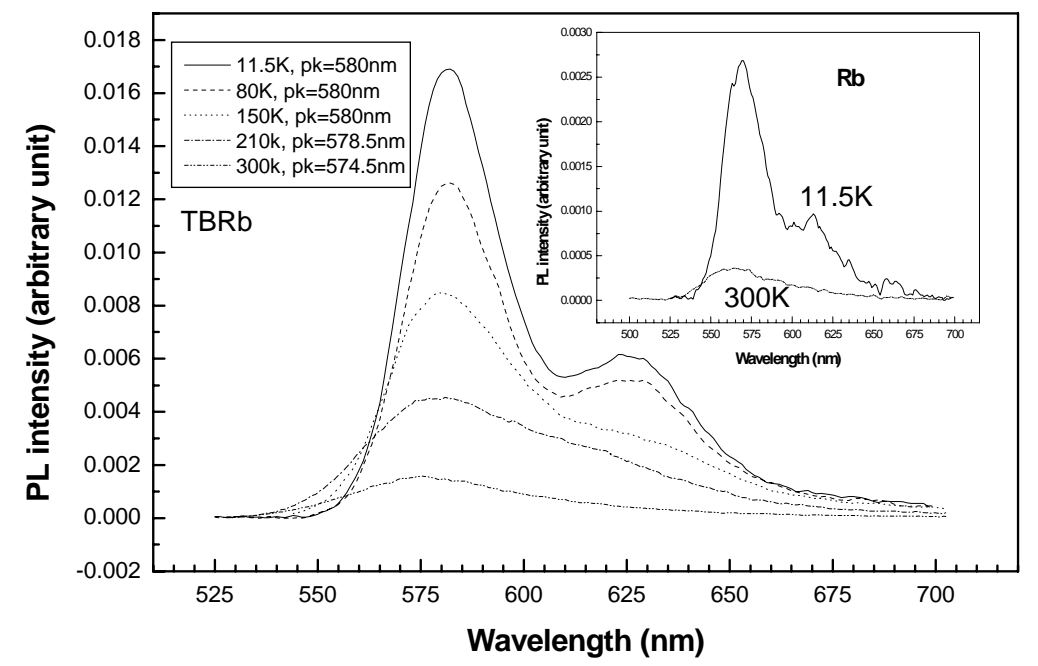

Figure 3 


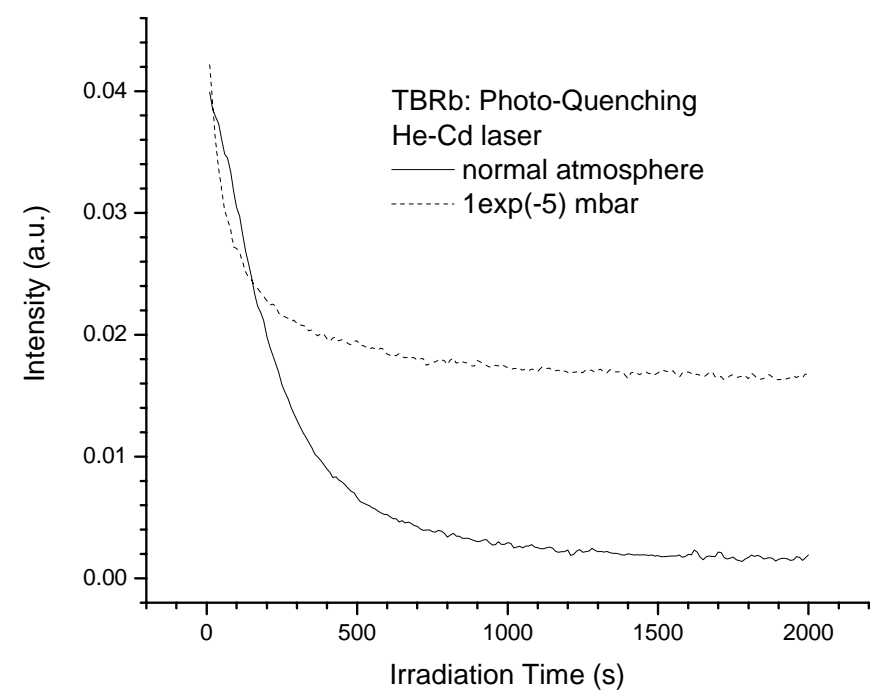

Figure 4 


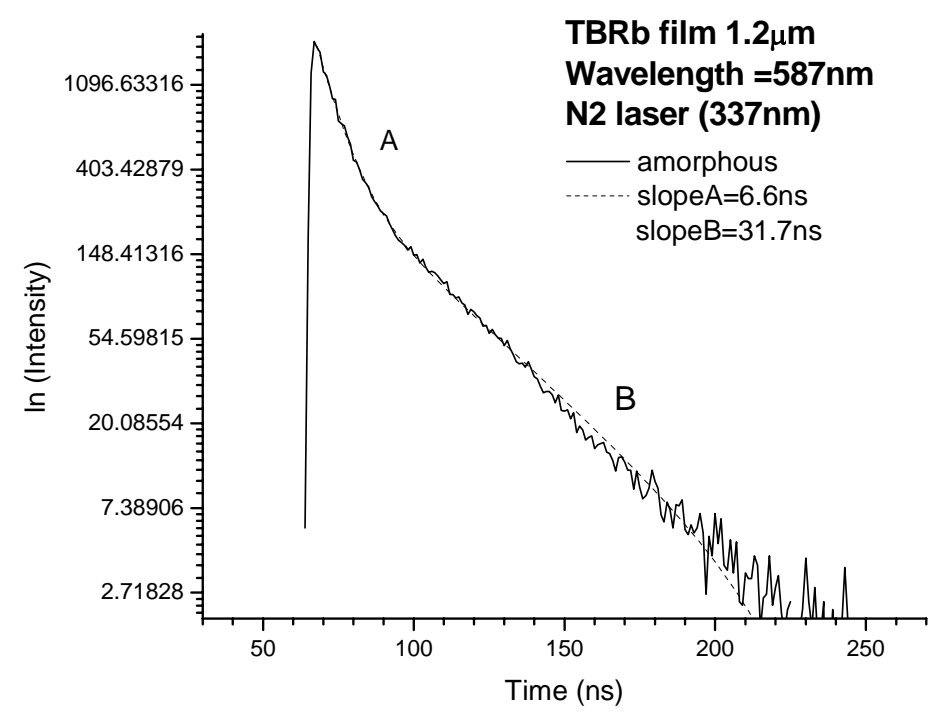

Figure 5 


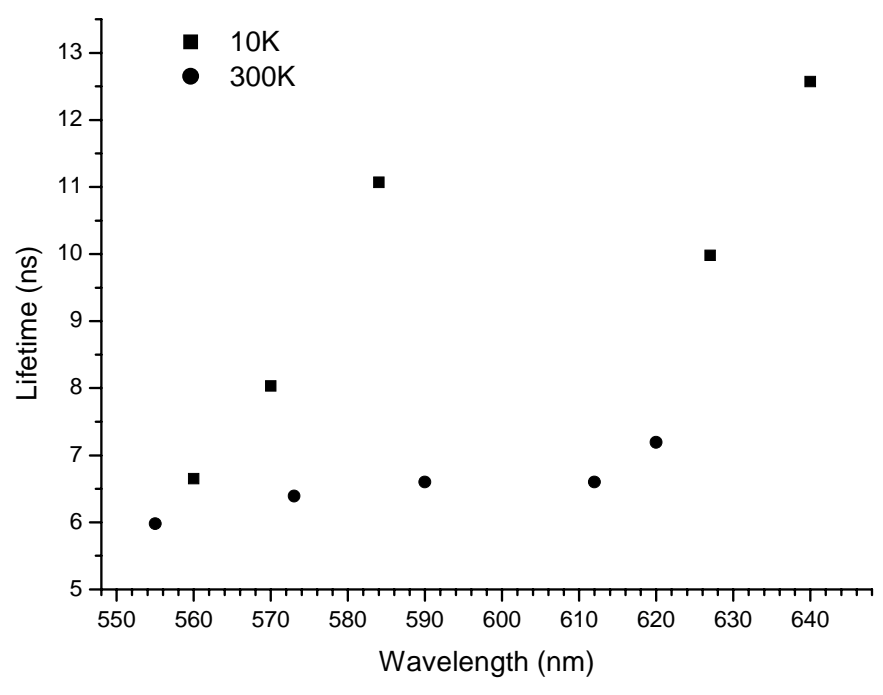

Figure 6 


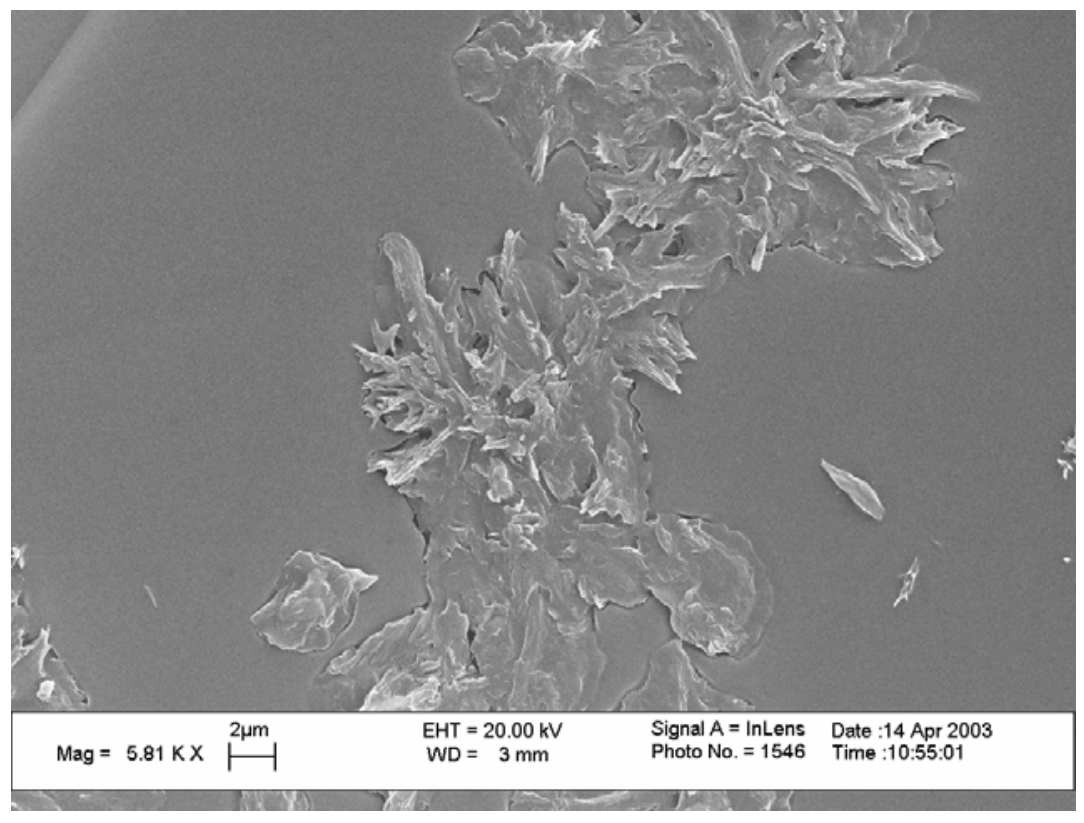

Figure 7a

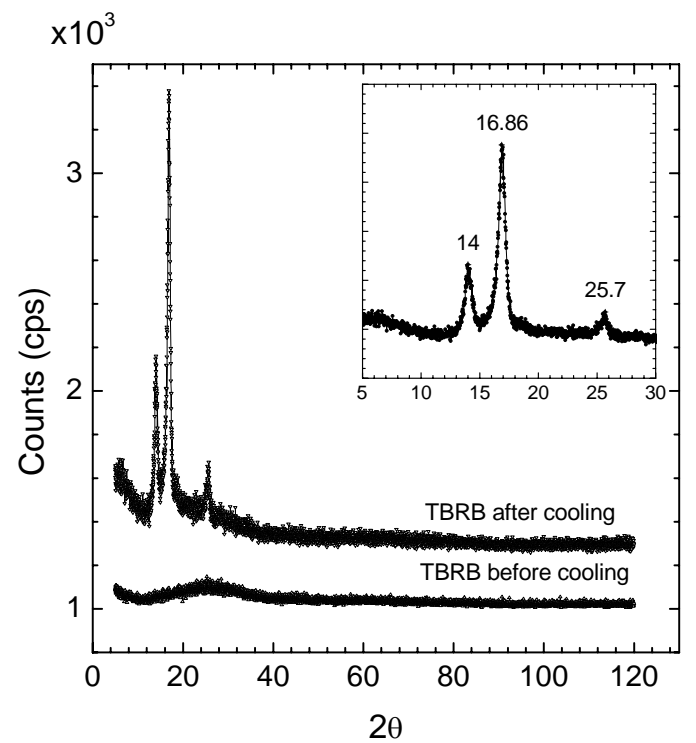

Figure 7b 